\title{
Visualizing and evaluating mitochondrial cysteine via near-infrared fluorescence imaging in cells, tissues and in vivo under hypoxia/ reperfusion stress
}

\author{
Xia Zhang ${ }^{\text {a,e }}$, Liangwei Zhang ${ }^{\text {a,c }}$, Xiaoyan Wang ${ }^{\text {a,d }}$, Xiaoyue Han ${ }^{\text {a,e }}$, Yan Huang ${ }^{\text {a,d }}$, Bowei Li ${ }^{\text {a,c }}$, \\ Lingxin Chen ${ }^{a, b, c, "}$ \\ ${ }^{a}$ CAS Key Laboratory of Coastal Environmental Processes and Ecological Remediation, Shandong Key Laboratory of Coastal Environmental Processes, Yantai Institute of \\ Coastal Zone Research, Chinese Academy of Sciences, Yantai 264003, China \\ ${ }^{\mathrm{b}}$ Laboratory for Marine Biology and Biotechnology, Pilot National Laboratory for Marine Science and Technology, Qingdao 266237, China

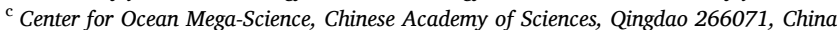 \\ d School of Pharmacy, Binzhou Medical University, Yantai 264003, China \\ ${ }^{\mathrm{e}}$ University of Chinese Academy of Sciences, Beijing 100049, China
}

\section{A R T I C L E I N F O}

\section{Editor: Dr. R Teresa}

\section{Keywords:}

Fluorescent probe

Cysteine

Near-infrared imaging

Hypoxia/reperfusion

Environmental stress

\begin{abstract}
A B S T R A C T
Increasingly grim environmental pollutions are closely related with the occurrence and development of diseases. However, it's obscure how environmental stress disturbs the normal physiological process, and then how endogenous reactive species mend the cases. Hypoxia/reperfusion (H/R), a common and intractable injury in aquaculture and clinic, can induce oxidative stress and ultimately cause irreversible injury to organism. Cysteine (Cys) plays essential roles in maintaining transduction of numerous reactive species and redox homeostasis in subcellular structures, cells and organisms. A great deal of fluorescence research about Cys are focusing on development of selective probes but with poor exploration of the biofunction under environmental stress. Therefore, it is of great significance to examine the bio-effects of Cys against H/R stress. In the present work, we design a fluorescent probe BCy-AC for in situ detecting Cys, the unique Enol-Keto tautomerization of fluorophore $\mathrm{BCy}$-Keto propels the reaction process which will improve the sensitivity and potential application performance of the probe. BCy-AC is conveniently applied to visualize Cys in HT-22 cells, zebrafish and mice tissues. Moreover, imaging results obtained from $\mathrm{H} / \mathrm{R}$ models reveal that endogenous Cys changes with hypoxia and reperfusion time and Cys pretreatment effectively defend H/R injury in cells and in vivo.
\end{abstract}

\section{Introduction}

A healthy ecological environment is the basis for human survival and development, however, the continuous circulation of environmental pollutants in atmosphere, water, and soil seriously threaten ecosystem safety and life health (Gavrilescu et al., 2015). These pollution issues may result in secondary environmental stress: hypoxia in air, soil and water, which posing danger to ecological sustainable development and human health (Petrie et al., 2015; Gray et al., 2002). As one pressing environmental problem, hypoxia environmental stress would gravely damage aquaculture industry and human health. Oxygen sufficiency is critical for organism oxygen homeostasis which involved in redox equilibrium of internal environment, otherwise, organism will undergo oxidative stress and outburst of reactive oxygen species (ROS), and then excessive accumulation of ROS in organism will further activate the antioxidant defense system (Jung et al., 2017; Wang et al., 2019; Cheng et al., 2017; Kim et al., 2015). Once ROS outburst and redox imbalance, it will also causes many other biological alterations, for instance, reactive sulfur species (RSS) mass production and signal transduction among numerous active species (Chen et al., 2011). Besides, hypoxia/reperfusion $(H / R)$ process could also initiate pernicious cellular responses and further inflict a cascade of damage in tissues, organ, and even threatening the health of the organism ultimately (Gao et al., 2019). This invasive process frequently occurs in environment hypoxia stress, some surgeries and diseases, such as ligation operation, inflammation, cancer, cerebral infarction, other cardiovascular and

\footnotetext{
* Corresponding author at: CAS Key Laboratory of Coastal Environmental Processes and Ecological Remediation, Shandong Key Laboratory of Coastal Environmental Processes, Yantai Institute of Coastal Zone Research, Chinese Academy of Sciences, Yantai 264003, China.

E-mail address: lxchen@yic.ac.cn (L. Chen).
} 
cerebrovascular diseases and central nervous system disorders. As a family of S-containing reactive species, cysteine (Cys), homocysteine (Hcy), and glutathione (GSH), etc, intracellular biothiols exhibits various biofunctions for maintaining cell homeostasis (Zhang et al., 2014; Guo et al., 2012). Despite the endogenous concentration is at micromole levels and the structures is similar with Hcy and GSH, Cys plays irreplaceable biological roles in various physiology and pathology activities (Zhang et al., 2019a; Yang et al., 2011; Ebisch et al., 2006). Not only Cys is the precursor in the synthesis of GSH and the product in the metabolism of Hcy, but also it participates in many biological events of protein synthesis, metabolism, detoxification and other physiological processes (Reddie and Carroll, 2008; Paulsen and Carroll, 2013).

Cys also exhibits reducibility and nucleophilic abilities, it's able to directly scavenge intracellular oxidants as well as involved in intricate generation and transformation of other RSS to struggle intracellular oxidative stress indirectly (Scheme 1) (Zhang et al., 2014, 2019a; Guo et al., 2012; Yang et al., 2011; Ebisch et al., 2006). However, abnormal intracellular Cys levels will result in many diseases syndromes including cardiovascular disease, neurotoxicity, liver damage, retarded growth, hair depigmentation, weakness and so on (Xue et al., 2015; Shahrokhian, 2001). Mitochondrion is indispensable highly dynamic cellular organelle, whose function is critical for maintaining cellular redox homeostasis and plays key roles in programed cell apoptosis (Jung et al., 2017; Gao et al., 2011; Rohlenova et al., 2017). In spite of the protective effects defended by intracellular antioxidant system, it is hard to avoid oxidative stress damage that result from uncontrolled ROS accumulation in mitochondria (Cheng et al., 2017; Han et al., 2018a; Sun et al., 2018; Guo et al., 2014). After decades of development, although plenty of researches about Cys including fluorescent probes have been investigated, most of them are short of noninvasive means for in situ detecting Cys in cells, tissues, or in vivo, no matter for real time evaluating bioeffects of Cys under environmental stress (Yang et al., 2011; Xue et al., 2015; Yin et al., 2017; Li et al., 2017; Zhang et al., 2015). Investigating mitochondria Cys fluctuation under hypoxic environmental stress is conducive to understanding the biological performance and making for finding effective means against environmental hypoxia stress.

The common methods for Cys detection basically include mass spectrometry (MS), high performance liquid chromatography (HPLC), HPLC-MS, and capillary electrophoresis separations methods (Guo et al., 2012; Zhang et al., 2019a). Although these methods have made significant contribution to Cys research, there generally need complicated pretreatment for samples and are not suitable for noninvasive detection. Near-infrared (NIR) fluorescent probes combined with confocal imaging techniques having unique advantages for tracing reactive molecules in living biosystems (Xu et al., 2015), since they can deeply penetrate into tissues and offer high spatial and temporal resolution, low auto-fluorescence background as well as less invasiveness (Sedgwick et al., 2018; Jung et al., 2013; Zhang et al., 2019b, 2020). Despite great advances have made in Cys fluorescence probes, it's also difficult to develop novel strategy and practical detection due to similar chemical structure and properties of Cys with other biological thiols. The previous reported Cys-specific fluorescence probes mainly focused on developing novel strategy of probe design or improving their selectivity and sensitivity but rarely referring exploration of Cys bioeffect induced by environmental stress, especially for hypoxia stress (Table S1). Focusing on hypoxia stress, we have developed two probes for exploring the protective roles of GSH as the work basis (Zhang et al., 2019b). Compared with the previous Cys probes, BCy-AC in this work showed NIR fluorescence spectra $\left(\lambda_{\mathrm{ex}} / \lambda_{\mathrm{em}}=648 / 726 \mathrm{~nm}\right)$ and unique Enol-Keto tautomerization which further boosted sensitivity and application via propelled reaction process. Therefore, it's imperative to develop a NIR florescent probe for discriminating and detecting mitochondria Cys as well as investigating and evaluating Cys biofunction under environmental hypoxia stress.

With these considerations in mind, we have devised a NIR fluorescent probe for in situ observing mitochondria Cys in cells, tissue, and in vivo under environmental $\mathrm{H} / \mathrm{R}$ stress. The probe BCy-AC was fabricated with an acrylate group (Cys recognition unit) and a NIR fluorophore BCy-Keto, which harvested in one step and owned mitochondria target ability. The unique Enol-Keto tautomerization of BCy-Keto propels the reaction process between $\mathrm{BCy}-\mathrm{AC}$ and analyte Cys (Scheme 2), and the thoroughness reaction of $\mathrm{BCy}-\mathrm{AC}$ assuredly improves the sensitivity and potential application performance for Cys. The stable ketone structure of BCy-Keto under simulated physiological condition facilitates the probe releasing fluorophore and turning on fluorescence when detecting Cys. Moreover, the NIR emission $(726 \mathrm{~nm}$ ) can noninvasively and deeply penetrate into tissue or in vivo. Using BCy-AC, we actually investigated Cys concentration changes in $\mathrm{H} / \mathrm{R}$ process and evaluated the connection between Cys concentrations and H/R-induced damage, and confirmed that Cys could play protective roles to defense environmental $\mathrm{H} / \mathrm{R}$ induced mitochondria related injury.

\section{Experimental section}

\subsection{Establishment of hypoxia/reperfusion cell models and zebrafish models}

To simulate hypoxia/reperfusion $(\mathrm{H} / \mathrm{R})$ process and establish cell $\mathrm{H}$ /

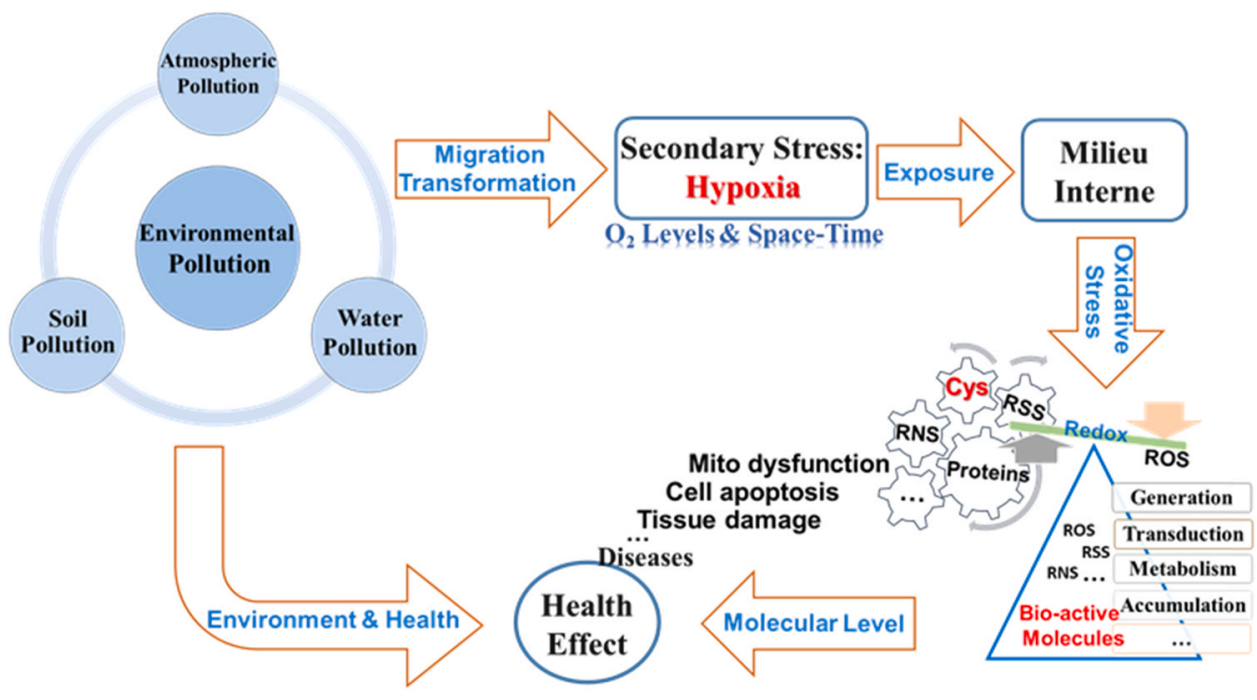

Scheme 1. Environmental pollution induced secondary hypoxia stress and proposed toxicology. 


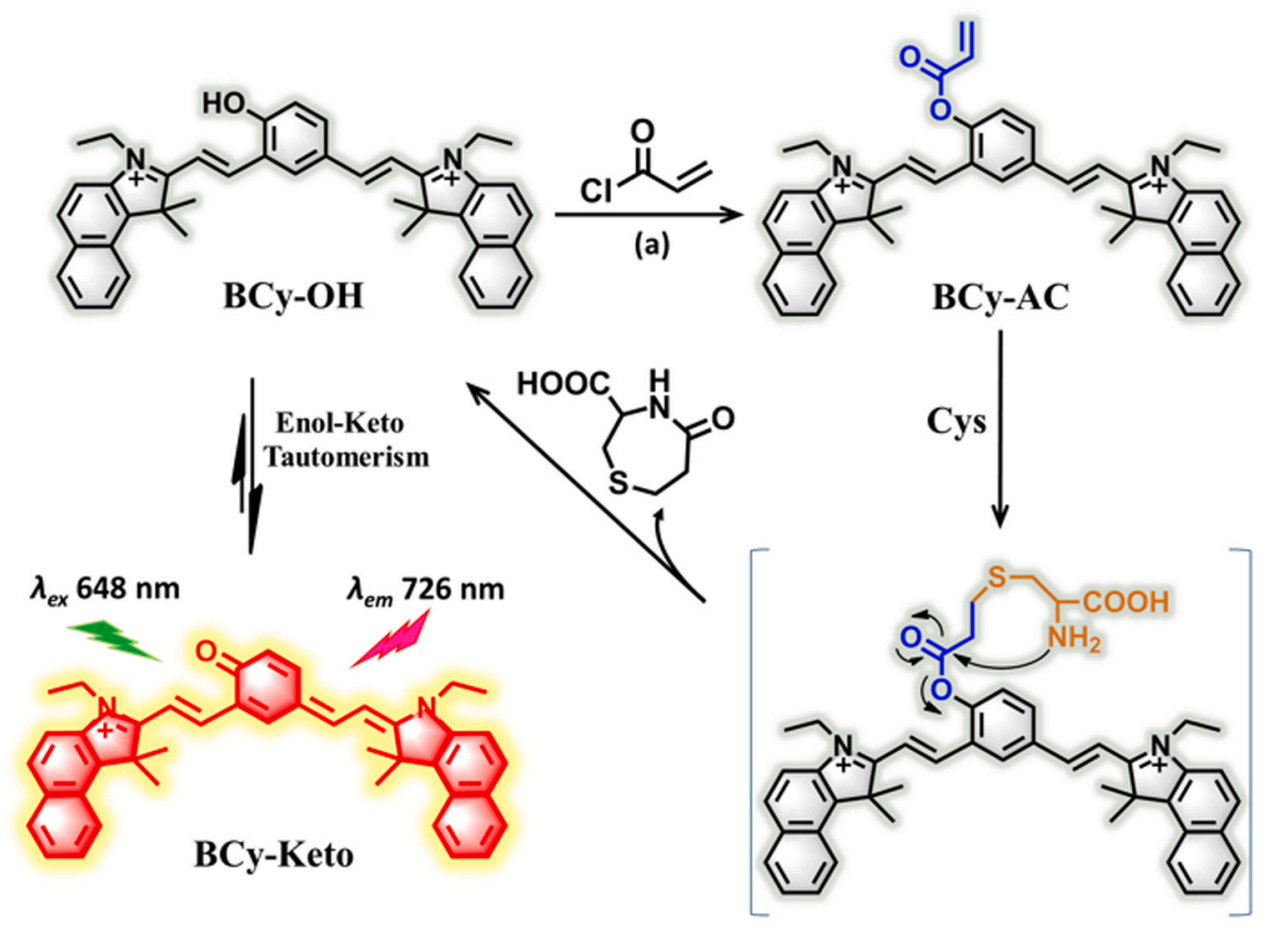

Scheme 2. Synthesis route of BCy-AC and proposed mechanism towards Cys.

R models, hippocampal neuronal cells (HT-22) were cultivated under $0.1 \% \mathrm{O}_{2}$ concentration via AnaeroPouch for $30 \mathrm{~min}$ and $60 \mathrm{~min}$, and then the normal $\mathrm{O}_{2}$ condition was recovered for $10 \mathrm{~min}$ and $30 \mathrm{~min}$ as reperfusion simulation after hypoxia stress. The cells in other groups were respectively pretreated with $0 \mu \mathrm{M}, 100 \mu \mathrm{M}, 300 \mu \mathrm{M}$ Cys for $30 \mathrm{~min}$ before general $\mathrm{H} / \mathrm{R}$ operation, they were established to investigate and evaluate the relationship between Cys levels and mitochondrial related $\mathrm{H} / \mathrm{R}$ damage. To further explore the relationship between endogenous Cys and H/R stress, three days post fertilization (3dpf) zebrafish larvaes were suppressed cardiac contractility and attained cerebral anoxia via pretreating 15 mM 2,3-butanedione monoxime (BDM) (Liu et al., 2014; Jou et al., 2010). The larvaes of zebrafish were suppressed $0 \mathrm{~min}, 2 \mathrm{~min}$, $6 \mathrm{~min}$ for hypoxia and then underwent reperfusion for $10 \mathrm{~min}$ and $30 \mathrm{~min}$

\subsection{Construction of hypoxia/reperfusion models in mice}

BALB/c mice were placed in normobaric hypoxic chambers (BioSpherix OxyCycler A84XOV, USA) for 1 day (FIO2, fraction of inspiration $\mathrm{O}_{2}, 11 \%$ ) and then performed $2 \mathrm{~h}$ reperfusion to construct mild $\mathrm{H} / \mathrm{R}$ models $\left(+\right.$ ), and hypoxia for 2 days (FIO2, fraction of inspiration $\mathrm{O}_{2}$, $11 \%$ ) and underwent $2 \mathrm{~h}$ reperfusion to build severe $\mathrm{H} / \mathrm{R}$ models $(++)$ (Gao et al., 2018). The mice in Cys treatment groups were pretreated with $100 \mu \mathrm{g} / \mathrm{kg}$ Cys (in saline, intraperitoneal injection, twice daily) for 2 weeks before executed $\mathrm{H} / \mathrm{R}$ operation.

\subsection{Confocal laser scanning imaging}

Confocal laser scanning microscope (Olympus FV1000, Japan) with objective lens $(\times 60)$ was used to observe the fluorescence imaging of cells at different groups. The cells were firstly inoculated into Petridishes $(20 \mathrm{~mm})$ and adherently cultivated for $24 \mathrm{~h}$. Before imaging, the cells were incubated with $10 \mu \mathrm{M} \mathrm{BCy}$-AC for $10 \mathrm{~min}$ at $37^{\circ} \mathrm{C}$. The parameters of commercial dyes were set according to their instructions. The colocalization parameters analysis of the co-stained cells was obtained by Image Pro Plus software. Fluorescence imaging of endogenous Cys levels in zebrafish larvaes and mice hippocampi slices were also obtained via confocal laser scanning microscope. At $28{ }^{\circ} \mathrm{C}$, the $3 \mathrm{dpf}$ zebrafish larvaes were pretreated with $10 \mu \mathrm{M}$ BCy-AC for $10 \mathrm{~min}$ and then performed in situ imaging through an $(\times 4)$ objective lens. The larvaes anesthetized by $35 \mathrm{mg} / \mathrm{L}$ tricaine solution for $5 \mathrm{~min}$ (Ravichandiran et al., 2021a, 2020). The hippocampi slices of mice were quickly obtained and fixed from the mice H/R models. After finished paraffin section dewaxing, the slices stained with $10 \mu \mathrm{M}$ probe at $37{ }^{\circ} \mathrm{C}$ for $10 \mathrm{~min}$ and observed imaging by the microscope with an $(\times 10)$ objective lens.

\subsection{In situ fluorescence imaging in mice}

Fluorescence imaging of Cys in mice were in situ obtained through PerkinElmer IVIS Lumina XRMS Series III (Perkinelmer, USA). The mice were intraperitoneal (i.p.) injected BCy-AC $(10 \mu \mathrm{M}, 100 \mu \mathrm{L}$ in DMSO/ saline, $\mathrm{v} / \mathrm{v}$ 1:99) for $15 \mathrm{~min}$ before imaging. To observe the imaging ability of Cys by the probe, the mice were pretreated with different thiols. The control group was only injected with probe for imaging, all the tested groups were consisted of 6 mice in parallel. The mice in $\mathrm{N}$ ethylmaleimide (NEM, a thiol-blocking agent) group were treated with NEM $(250 \mu \mathrm{g} / \mathrm{kg}, 1 \mathrm{~h})$ and then imaging Cys through BCy-AC. The mice in Cys groups were first treated with NEM and then injected Cys $(100 \mu \mathrm{M}, 200 \mu \mathrm{L})$, Cys $(300 \mu \mathrm{M}, 200 \mu \mathrm{L})$ respectively for $30 \mathrm{~min}$ before $\mathrm{BCy}$-AC imaging. Hcy group was treated with NEM and then incubated Hcy $(300 \mu \mathrm{M}, 200 \mu \mathrm{L})$ for $30 \mathrm{~min}$ before imaging. Chloral hydrate $(400 \mathrm{mg} / \mathrm{kg}$, i.p.) was used to anesthetize the mice and isoflurane (2\%) was utilized to keep anesthesia.

\subsection{Synthetic procedure of the probe BCy-AC}

The synthesized procedure of BCy-AC was displayed in Scheme 2. BCy-Keto was prepared following our previous work (Zhang et al., 2019b). Acryloyl chloride $(0.16 \mathrm{~mL}, 2 \mathrm{mmol})$ was dissolved in $10 \mathrm{~mL}$ $\mathrm{CH}_{2} \mathrm{Cl}_{2}$ under argon atmosphere at $0{ }^{\circ} \mathrm{C}$, and then few N-Ethyldiisopropylamine was instilled into the solution for $30 \mathrm{~min}$ stirring. $10 \mathrm{~mL} \mathrm{CH}_{2} \mathrm{Cl}_{2}$ containing BCy-Keto $(177 \mathrm{mg}, 0.3 \mathrm{mmol}$ ) was dropwise instilled into above solution system and lasted for $1 \mathrm{~h}$ at $0{ }^{\circ} \mathrm{C}$, after that, 
the reaction was reacted overnight under ambient temperature. The reaction product was extracted by $\mathrm{CH}_{2} \mathrm{Cl}_{2}$ from the aqueous media and then the organic phase was concentrated under reduced pressure. The crude product was purified through silica chromatography (200-300 mush) with methanol and ethyl acetate, BCy-AC was obtained, claybank solid (64.1\%). ${ }^{1} \mathrm{H}$ NMR (500 MHz, $\mathrm{CDCl}_{3}-\mathrm{d}_{1}$ ) $\delta(\mathrm{ppm})$ : 8.10-8.09 (m, $1 \mathrm{H})$, 7.94-7.93 (m, 2H), 7.87-7.83 (m, 4H), 7.72-7.71 (m, 3H), 7.54-7.52 (m, 6H), 7.37-7.35 (m, 2H), 7.23-7.22 (s, 2H), 5.36-5.34 (t, $2 \mathrm{H}), 3.90-3.86$ (q, 4H), $1.33-1.25(\mathrm{~m}, 18 \mathrm{H}) .{ }^{13} \mathrm{C}$ NMR $(125 \mathrm{MHz}$, $\left.\mathrm{CDCl}_{3}-\mathrm{d}_{1}\right) \delta(\mathrm{ppm}): 182.2,167.8,166.1,139.1,132.4,131.2,131.0$, 130.5, 129.9, 129.7, 129.5, 129.0, 128.9, 128.2, 127.4, 126.9, 123.4, 122.1, 109.8, 65.6, 45.8, 24.0, 13.8. LC-MS $\left(\mathrm{ESI}^{+}\right): \mathrm{m} / \mathrm{z} \mathrm{C}_{45} \mathrm{H}_{44} \mathrm{~N}_{2} \mathrm{O}_{2}^{2+}$ calcd. 322.17 , found $\left[\mathrm{M}^{2+}\right] 322.56$.

\subsection{Ethics statement}

All procedures and surgical operations conformed to the National Guidelines for the Care and Use of Laboratory Animals. This work has received approval for research ethics from Binzhou Medical University and a proof/certificate of approval is available upon request (Approval Number: No. IRB2021-08).

\section{Results and discussion}

\subsection{Spectra of BCy-AC for Cys}

With the probe in hand, the sensing ability of BCy-AC for Cys was examined by UV-vis spectral in HEPES buffer (10 mM, pH 7.4) initially. As shown in Fig. 1a, the characteristic peaks of BCy-Keto gradually appeared and enhanced while BCy-AC given no obvious absorption peak at $400-800 \mathrm{~nm}$ with increasing Cys concentration $(0-20 \mu \mathrm{M}$ ) (Zhang et al., 2019b). Simultaneously, the fluorescence spectra also exhibited dose-dependent enhancement at $726 \mathrm{~nm}\left(\lambda_{\mathrm{ex}}=648 \mathrm{~nm}\right.$, Fig. 1b), this "turn-on" fluorescence response existed good linear relationship between BCy-AC and Cys (Fig. 1c, $\mathrm{F}_{726}=29.7508$ [Cys] + 36.4158, $\mathrm{R}^{2}$ $=0.9893,0-20 \mu \mathrm{M})$. The limit of detection was calculated to be as low as $0.15 \mu \mathrm{M}(3 \sigma / k)$, which was much lower than the endogenous Cys levels in cells (30-200 $\mu \mathrm{M})$ (Cai et al., 2020). For selectivity, the potential interference species were respectively verified by BCy-AC under same testing conditions in Fig. 1d. The results displayed that only Cys caused obvious fluorescence enhancement while other amino acids and reactive species caused negligible fluorescence signal. Moreover, the sensing behavior and time-depended response of BCy-AC for Cys, Hcy, and GSH were also measured (Fig. 1e-f). Although the structure and reactivity of Hcy and GSH were similar to Cys, the spectra of these two thiols displayed quite a little of disparity from that of cysteine. This could attribute to the reaction intermediates had difficulty in intramolecular cyclizations after BCy-AC underwent nucleophilic reaction with Hcy and GSH (Scheme S1). However, the formation of seven-membered ring in the intermediate induced by Cys was more favorable to intramolecular cyclization than that of eight-membered ring induced by Hcy in dynamics (Guo et al., 2012; Zhang et al., 2019a). Thus the fluorescence sensing behaviors of BCy-AC for Cys and Hcy were different under same handling time. As for GSH, stereo-hindrance effect of its structure hindered intramolecular cyclization of intermediate, which resulted in the probe rest on conjugated thioether with feeble fluorescence response (Jung et al., 2012).

\subsection{In situ imaging of intracellular Cys and sub-location of the probe}

Encouraged by above results and combined with characteristics of NIR dyes, the bioimaging capacity of BCy-AC was further examined in living systems. At first, the biocompatibility of probe was assessed via MTT assays and high cells viability demonstrated low cytotoxicity of BCy-AC in living cells and high feasibility in bioresearch (Fig. S2). After that, the probe was applied to monitor endogenous and exogenous Cys in HT-22 cells (Fig. S3 and Fig. 2). The cells emitted modest fluorescence of endogenous Cys with incubation of probe for $10 \mathrm{~min}$ in Fig. 2a, the cells pretreated with $200 \mu \mathrm{M}$ NEM exhibited more feeble fluorescence in Fig. 2b. As shown in Fig. 2e, after incubation with NEM and $300 \mu \mathrm{M}$ Cys, the fluorescence intensity was enhanced when the cells stained with $\mathrm{BCy}-\mathrm{AC}$ for $10 \mathrm{~min}$. The time-dependent fluorescence changes
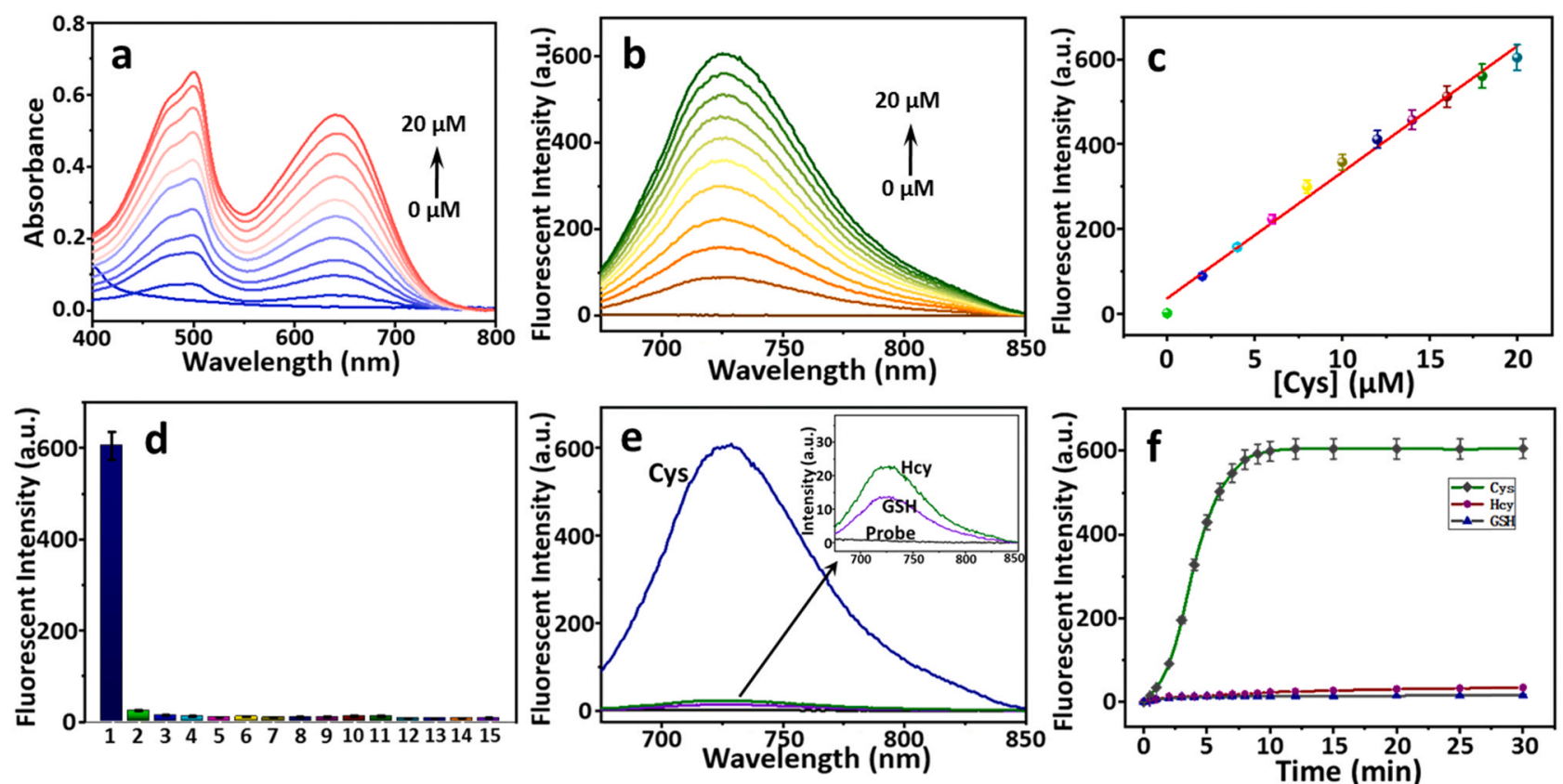

Fig. 1. Spectral studies and selectivity of BCy-AC for cysteine in $10 \mathrm{mM}$ HEPES buffer (pH 7.4, 1\% DMSO). (a) Dose-dependent UV-vis absorption and (b) Fluorescence spectra $\left(\lambda_{\text {ex }}=648 \mathrm{~nm}\right)$ of BCy-AC $(10 \mu \mathrm{M})$ towards Cys $(0-20 \mu \mathrm{M})$ at $37^{\circ} \mathrm{C}$ for $10 \mathrm{~min}$ (c) The linear relationship between fluorescence intensity (726 nm) and Cys concentration. (d) Selectivity of probe $(10 \mu \mathrm{M})$ for various biologically reactive species (2 equiv.). 1, Cys; 2, Hcy; 3, GSH (1 mM); 4, $\mathrm{H}_{2} \mathrm{~S} ; 5$, Gly; 6, Ser; 7, Glu; 8. Lys; 9, Ala; $10, \mathrm{H}_{2} \mathrm{O}_{2} ; 11, \mathrm{ClO}^{-} ; 12, \mathrm{Ca}^{2+} ; 13, \mathrm{Fe}^{2+} ; 14, \mathrm{Cu}^{2+} ; 15, \mathrm{Z}_{\mathrm{n} 2}^{+}$. (e) Fluorescence spectra of probe $(10 \mu \mathrm{M})$ in the presence of $\mathrm{Cys}(20 \mu \mathrm{M}), \mathrm{Hcy}(20 \mu \mathrm{M})$, and GSH $(50 \mu \mathrm{M})$ for $10 \mathrm{~min}$ (f) Time-dependent fluorescence intensity of BCy-AC upon addition of Cys $(20 \mu \mathrm{M})$, Hcy $(20 \mu \mathrm{M})$, and GSH $(50 \mu \mathrm{M})$. 

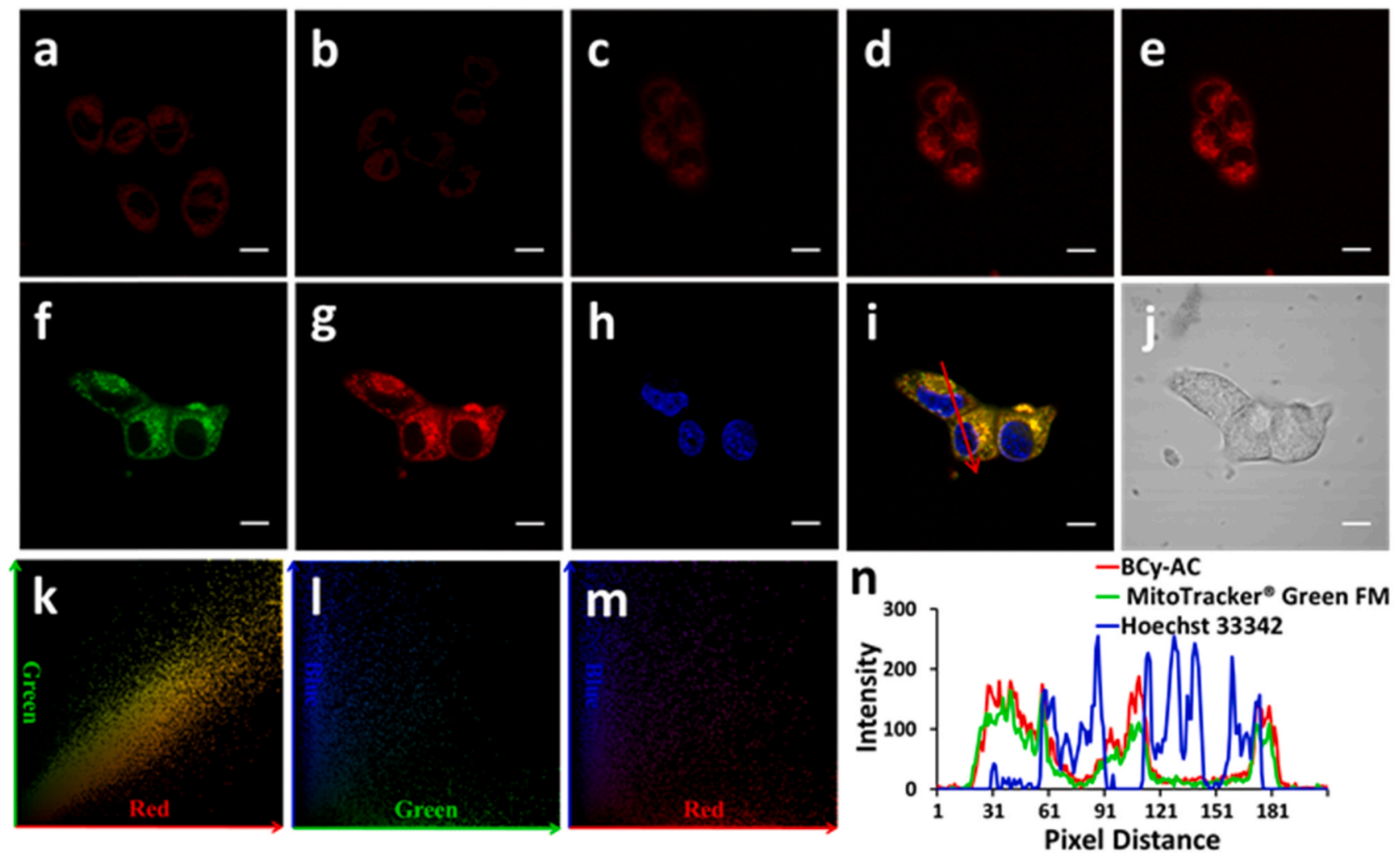

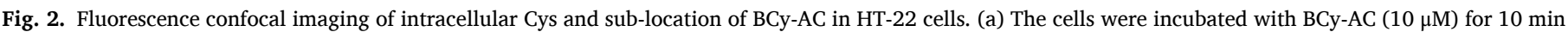

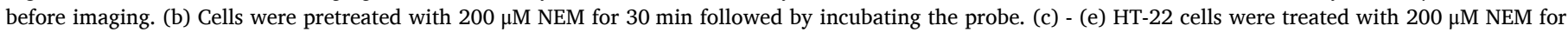

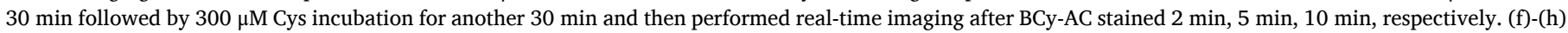

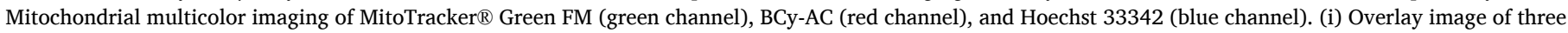

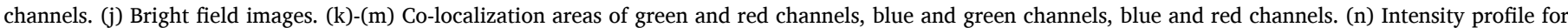

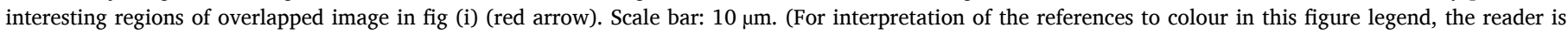
referred to the web version of this article.)

illustrated the response process of the probe for intracellular Cys was fast and completed within 10 min (Fig. 2c-e and Fig. S4). Furthermore, the quenching and increasing fluorescence in cells induced by NEM and exogenous Cys demonstrated that the probe was sensitive for real time imaging intracellular Cys.

To identify the distribution of BCy-AC in mitochondria, confocal microscopy imaging and fluorescence co-localization experiments were carried out using the probe and commercial dyes. First, the HT-22 cells were co-stained by BCy-AC (red channel, $10 \mu \mathrm{M}, 10 \mathrm{~min}$ ), commercial mitochondrial tracking dye MitoTracker ${ }^{\circledR}$ Green FM (green channel, $1 \mu \mathrm{g} / \mathrm{mL}, 15 \mathrm{~min}$ ), and nuclear dye Hoechst 33342 (blue channel, $1 \mu \mathrm{g}$ / $\mathrm{mL}, 30 \mathrm{~min}$ ). In Fig. 2f-h, the pseudo-color images of tested cells were collected from three channels: green $\left(\lambda_{\mathrm{em} / \mathrm{ex}}=500-580 \mathrm{~nm} / 488 \mathrm{~nm}\right)$, red $\left(\lambda_{\mathrm{em} / \mathrm{ex}}=700-800 \mathrm{~nm} / 635 \mathrm{~nm}\right)$, and blue $\left(\lambda_{\mathrm{em} / \mathrm{ex}}=420-480 \mathrm{~nm} /\right.$ $405 \mathrm{~nm}$ ). Fig. 2k-m presented the probe BCy-AC overlaid well with MitoTracker ${ }^{\circledR}$ Green FM and possessed desirable Pearsons's correlation coefficient between red and green channels, $\operatorname{Rr}_{(\mathrm{G}-\mathrm{R})}=0.957\left(\mathrm{Rr}_{(\mathrm{B}-}\right.$ $\left.\mathrm{G})=0.17, \operatorname{Rr}_{(\mathrm{B}-\mathrm{R})}=0.18\right)$. Moreover, $\mathrm{BCy}-\mathrm{AC}$ and mitochondrial tracking dye also revealed high anastomosis in the intensity profile analysis (Fig. 2n) of interesting regions in overlapped image (red arrow of Fig. 2i). The results of confocal fluorescence imaging and co-staining experiments confirmed that the probe BCy-AC was capable of penetrating into cells and distributing in mitochondria for recognizably sensing Cys quickly.

\subsection{Endogenous Cys changes and cellular damage under $H / R$ stress}

In order to further investigate the potential application of BCy-AC for endogenous Cys, cell $\mathrm{H} / \mathrm{R}$ models were established to visualize intracellular Cys fluctuation and evaluate the cell damages during the process. H/R models of HT-22 cells were designed as follow, the cells were incubated under $0.1 \% \mathrm{O}_{2}$ hypoxia for $0 \mathrm{~min}, 30 \mathrm{~min}, 60 \mathrm{~min}$, and then reperfusion for $10 \mathrm{~min}, 30 \mathrm{~min}$ (Fig. 3). The cell medium of hypoxia groups were deoxygenization beforehand, and then the cells were recovered normal oxygen supply timely when the hypoxia operations were finished. All the tested cells were incubated with $10 \mu \mathrm{M}$ BCy-AC before imaging and the fluorescence images of endogenous Cys in Fig. 3A-B. Compared with group a, the fluorescence signal in group b and group $\mathrm{c}$ increased slightly which revealed the levels of endogenous Cys rised tenderly along with the hypoxia time in HT-22 cells. However, the recovery of normal oxygen supply induced recession in fluorescence at different reperfusion time (Group d-e), revealing endogenous Cys significantly down-regulated at the early stage of reperfusion which may be probably closely related to cell damage. In Fig. 3C, the flow cytometry analysis (FCA) via BCy-AC intuitively reflected the changes of endogenous Cys in the H/R cells, and this Cys fluctuation trend also had been verified by the reported probe in Fig. S5 (Zhang et al., 2019a). The data in Fig. 3D showed that the apoptosis slowly changes in hypoxia phase while the states of damage became more severe in reperfusion phase. At hypoxia stages and long reperfusion stage, the up-regulation of mitochondria Cys may be related to cell auto-regulation. At the early stage of reperfusion, not only the endogenous Cys down-regulated obviously, but also more serious damaged occurred in the cells. This may be attributed to the consumption of mitochondria Cys by the burst of ROS during the reperfusion process (Gray et al., 2002; Paulsen and Carroll, 2013). Therefore, exogenous pre-incubation cells with Cys for preventing the decline of Cys at this stage may well be an effective prevention mean for $\mathrm{H} / \mathrm{R}$ damage. 


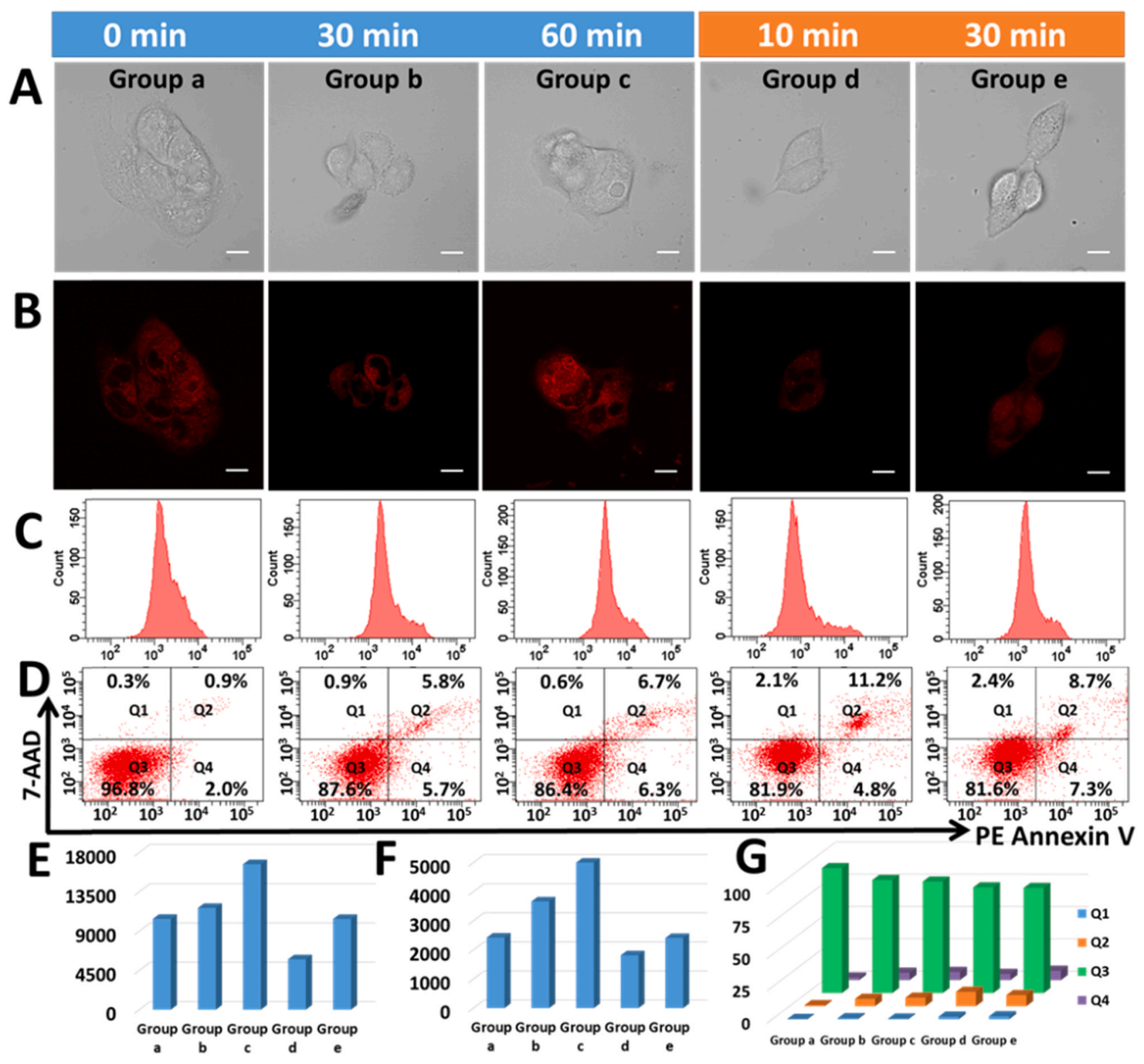

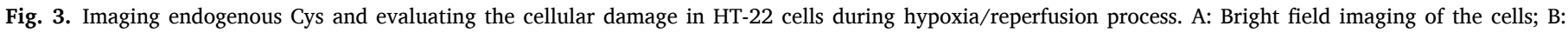

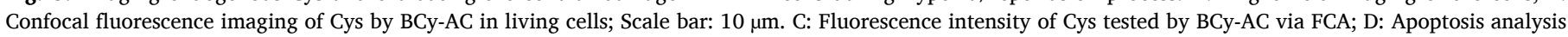

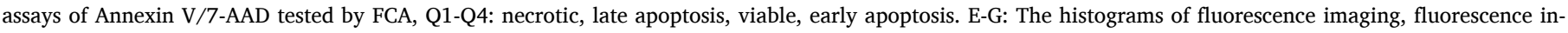

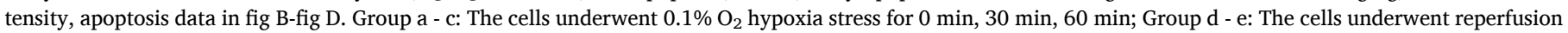
process for $10 \mathrm{~min}, 30 \mathrm{~min}$ after hypoxia stress. (BCy-AC: $\lambda_{\mathrm{em}}=700-800 \mathrm{~nm}, \lambda_{\mathrm{ex}}=635 \mathrm{~nm}$ ).

\subsection{Assessment Cys effects on $H / R$ induced cellular damage}

Since the fluctuation of Cys related with H/R-induced cellular damage, ROS as an essential participant of oxidative stress were determined through fluorescent probe Hydro-IR-783 to further reveal the effects of Cys and the H/R injury mechanism (Kundu et al., 2009). As shown in Fig. 4A, the fluorescence signals were weak and fluctuated slightly in H/R-free groups (group a-c), indicating that the impacts of exogenous Cys on ROS were negligible. In contrast, the significant fluorescence enhancement in H/R group (group d) was observed, confirming that ROS burst in HT-22 cells under H/R stress. Interestingly, the fluorescence signals of ROS were down-regulated with supplement of Cys concentration in $\mathrm{H} / \mathrm{R}$ models (group $\mathrm{d}-\mathrm{f}$ ). These results preliminarily demonstrated that pretreatment with Cys could affect intracellular ROS levels on H/R environmental stress. To confirm Cys in connection with cell oxidative damage induced by $\mathrm{H} / \mathrm{R}$ stress, FCA was used to examine damage parameters of the cells in Fig. 4B-C. PE-Annexin V/7-AAD analysis exhibited low apoptosis levels in $\mathrm{H} / \mathrm{R}$-free groups (Fig. 4B group a-c) while apoptosis was aggravated signally in $H / R$ groups. And the degree of apoptosis emerged gradual improvement when the cells were incubated with $100 \mu \mathrm{M}$ and $300 \mu \mathrm{M}$ Cys. Mitochondrial membrane potential $\left(\Delta \Psi_{m}\right)$ is one biomarker of cell early apoptosis, 5,5',6,6'-tetrachloro-1, $1^{\prime}, 3,3^{\prime}$ - tetraethyl-imidacarbocyanine (JC-1) was usually used for analyzing $\Delta \Psi_{m}$ via FCA. The red/green fluorescence ratio of JC-1 was depended on $\Delta \Psi_{m}$. The lower ratio, the lower $\Delta \Psi_{m}$, and the more serious apoptosis. In Fig. 4C, the downtrend of $\triangle \Psi_{m}$ was consistent with above apoptosis results, group d depicted lowest red/green ratio while group e-f present partial remission due to Cys treatments. Intracellular ROS was down-regulated, apoptosis and $\triangle \Psi m$ were improved from group $\mathrm{d}$ to group $\mathrm{f}$, which revealed that oxidative damage was gradually depressed when the cells pretreated with $0-300 \mu \mathrm{M}$ Cys on H/R stress.

Mitochondria are the vital place of complicated redox reaction as 


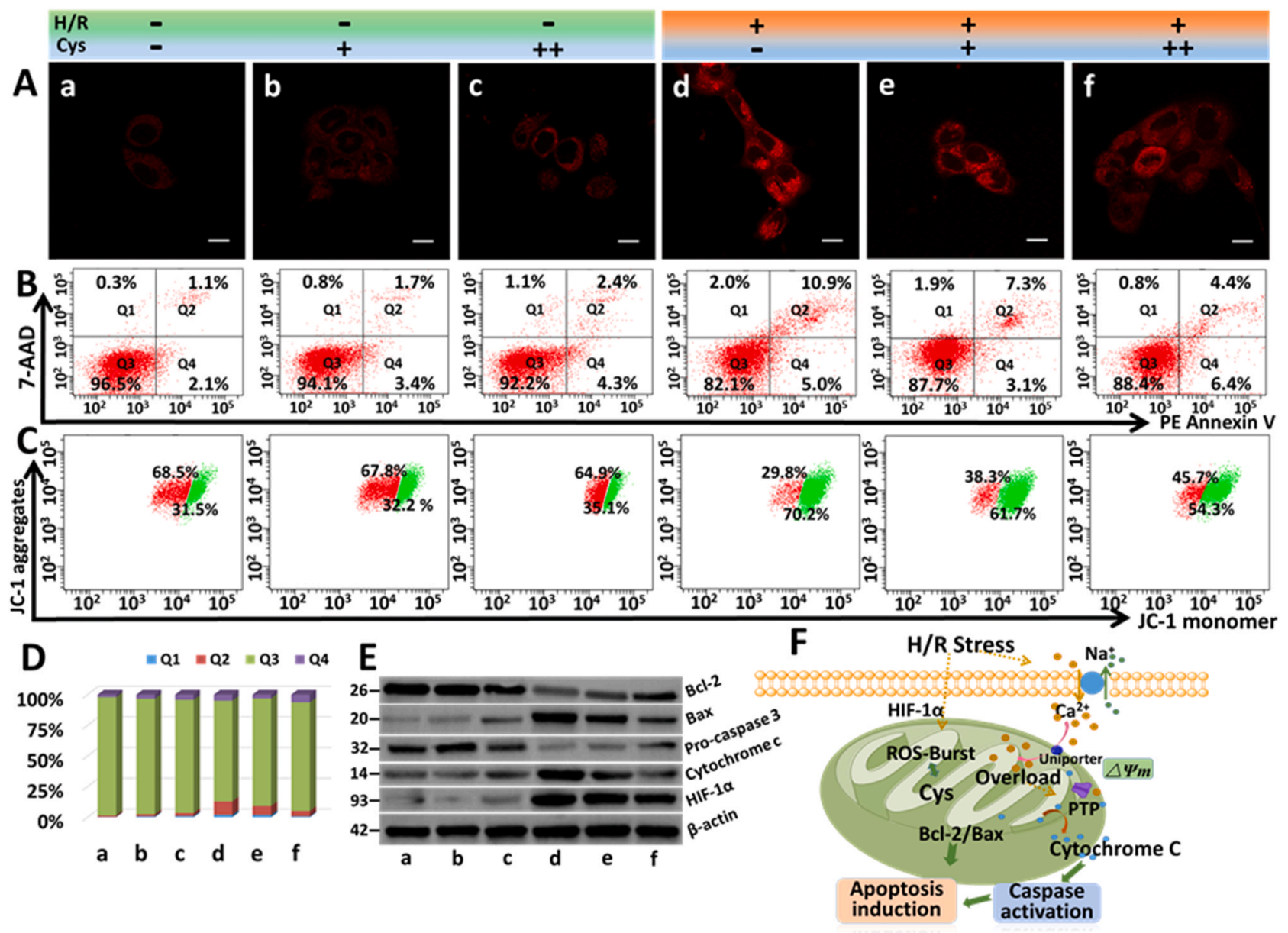

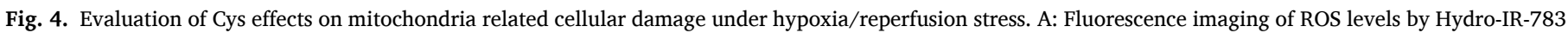

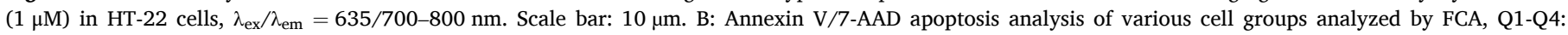

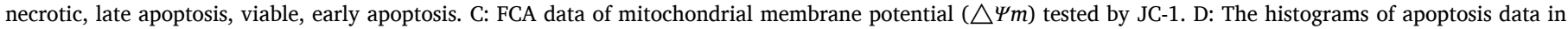

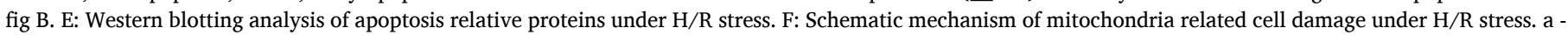

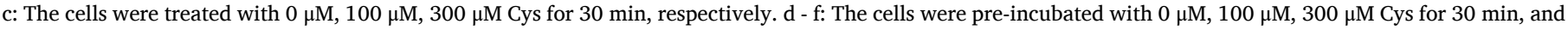
then executed $\mathrm{H} / \mathrm{R}$ operation (60 $\mathrm{min} / 10 \mathrm{~min})$.

well as the main production sites of ROS (Gao et al., 2011; Rohlenova et al., 2017; Won et al., 2015; Ravichandiran et al., 2021b). Cells H/R stress created excessive ROS in mitochondria and accumulation of ROS might led changes of downstream signal proteins Bcl-2 and Bax. Cytochrome $\mathrm{c}$ was the downstream effector protein of Bax and Bcl-2 and located in mitochondrial outer membrane participating in other biosignal transductions (Han et al., 2018b). Once the cells subjected severe oxidative stress, $\triangle \Psi_{m}$ would collapse and increasing ratio of Bax/Bcl-2 would assist the release of cytochrome $\mathrm{c}$ from mitochondria to cytoplasm, and then cytoplasm cytochrome c would activate the apoptotic executor cleaved-caspase 3 (Fig. 4E-F) (Porter and Jänicke, 1999). Western blotting analysis of relevant proteins was demonstrated in Fig. 4E, they displayed that the cells in $\mathrm{H} / \mathrm{R}$ group given high expression of hypoxia inducible factor (HIF-1 $\alpha$ ) and abnormal expression of caspase-dependent relevant apoptosis proteins. However, the abnormal expression for the several proteins gradually improved from group $d$ to group $f$ when the cells pre-incubated Cys on H/R process. H/R stress might greatly hinder the redox homeostasis, amount of ROS accumulated in the mitochondria of cells and then induced caspase-dependent cell apoptosis. On the one hand, as antioxidant, Cys may directly consume ROS and protect cells against H/R-induced cell damage; On the other hand, Cys act as signal transduction molecule may mediate other RSS to participate in redox regulation under H/R stress (Zhang et al., 2020; Kimura, 2019, 2014; Liu et al., 2019). The exogenous supplement of Cys could directly or indirectly consume the excessive ROS to weaken the levels of oxidative stress and achieve the protective effects for cells under $\mathrm{H} / \mathrm{R}$ environmental stress.

\subsection{The ability of BCy-AC for examining Cys in tissues and in vivo}

In view of excellent results in vitro, the $\mathrm{H} / \mathrm{R}$ models of $3 \mathrm{dpf}$ zebrafish larvaes were established through BDM (a cardiac contractility agent can attain cerebral anoxia) for further investigation of Cys fluctuation in vivo under H/R stress (Liu et al., 2014; Jou et al., 2010). As shown in Fig. 5, the zebrafish incubated with BCy-AC $(10 \mu \mathrm{M})$ for 10 min given no apparent signal. Incubation with BDM for $2 \mathrm{~min}$, the fluorescence appeared faintly, and then increasingly strengthened at $6 \mathrm{~min}$ after cardiac contractility suppression. The increasing fluorescence during zebrafish $\mathrm{H} / \mathrm{R}$ process revealed that the probe BCy-AC was capacity of in situ visualizing Cys timely and the larvaes could up-regulate Cys levels for resisting BDM-induced cerebral hypoxia stress. To examine Cys changes in reperfusion process, the residue BDM was removed to relieve suppression and anoxia effects (Liu et al., 2014; Jou et al., 2010). From group $\mathrm{d}$ to group e in Fig. 5A-B, the fluorescence of Cys gradually receded in head and epigastrium of the larvae along with the reperfusion time (within $30 \mathrm{~min}$ ), which might attribute to the generation of intracellular ROS gradually consumed the up-regulated Cys in zebrafish during reperfusion stress. The three-dimensional (3D) fluorescence 


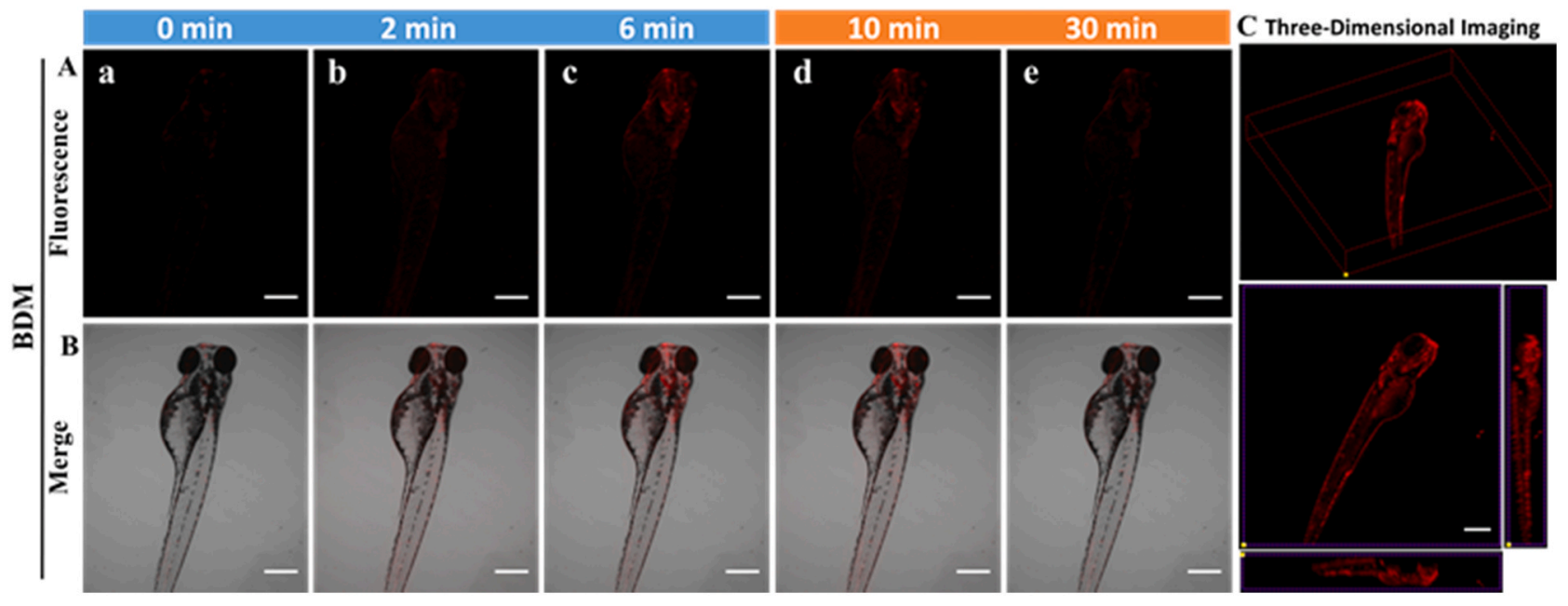

Fig. 5. In situ fluorescence imaging of zebrafish larvaes with $B C y-A C(10 \mu \mathrm{M})$ for endogenous Cys. A: Confocal laser scanning images of $H / R$ zebrafish models induced by BDM. B: Merge imaging of bright field and fluorescence. Group a - c: BDM induced zebrafish cerebral anoxia for $0 \mathrm{~min}, 2 \mathrm{~min}, 6 \mathrm{~min}$; Group d - e: The zebrafish then underwent reperfusion for $10 \mathrm{~min}, 30 \mathrm{~min}$ after hypoxia stress. C: Three-Dimensional fluorescence imaging of zebrafish larvae and representative slices of views from different directions, the zebrafish was pre-incubated $300 \mu \mathrm{M}$ Cys for $30 \mathrm{~min}$ at $28^{\circ} \mathrm{C}$. Scale bar: $30 \mu \mathrm{m}$. The data were shown as mean ( \pm s.d.) $(\mathrm{n}=6)$. BCy-AC: $\lambda_{\mathrm{em}}=700-800 \mathrm{~nm}, \lambda_{\mathrm{ex}}=635 \mathrm{~nm}$.

imaging of zebrafish in Fig. 5C exhibited bright fluorescence of Cys and thus positive results further suggested the application prospect of BCy-AC for in vivo imaging.

Fluorescence visualizing of Cys in peritoneal cavity of BALB/c mice via BCy-AC was further exhibited in Fig. 6A. The probe-loaded mice displayed weak fluorescence intensity while NEM-pretreated mice exhibited more feeble fluorescent signal in Fig. 6A (a) and (b), respectively. After sequentially pretreated with NEM, different amounts of Cys and the probe, the mice showed different degrees of fluorescence enhancement in, Fig. 6A (c) and (d). However, Hcy couldn't cause notable fluorescence change by probe imaging in Fig. 6A (e). These results illustrated the probe $\mathrm{BCy}-\mathrm{AC}$ was able to selectively detect Cys in mice and the NIR fluorescence characteristics of the probe achieved in situ visualizing Cys. Furthermore, the hippocampi slices of H/R mice

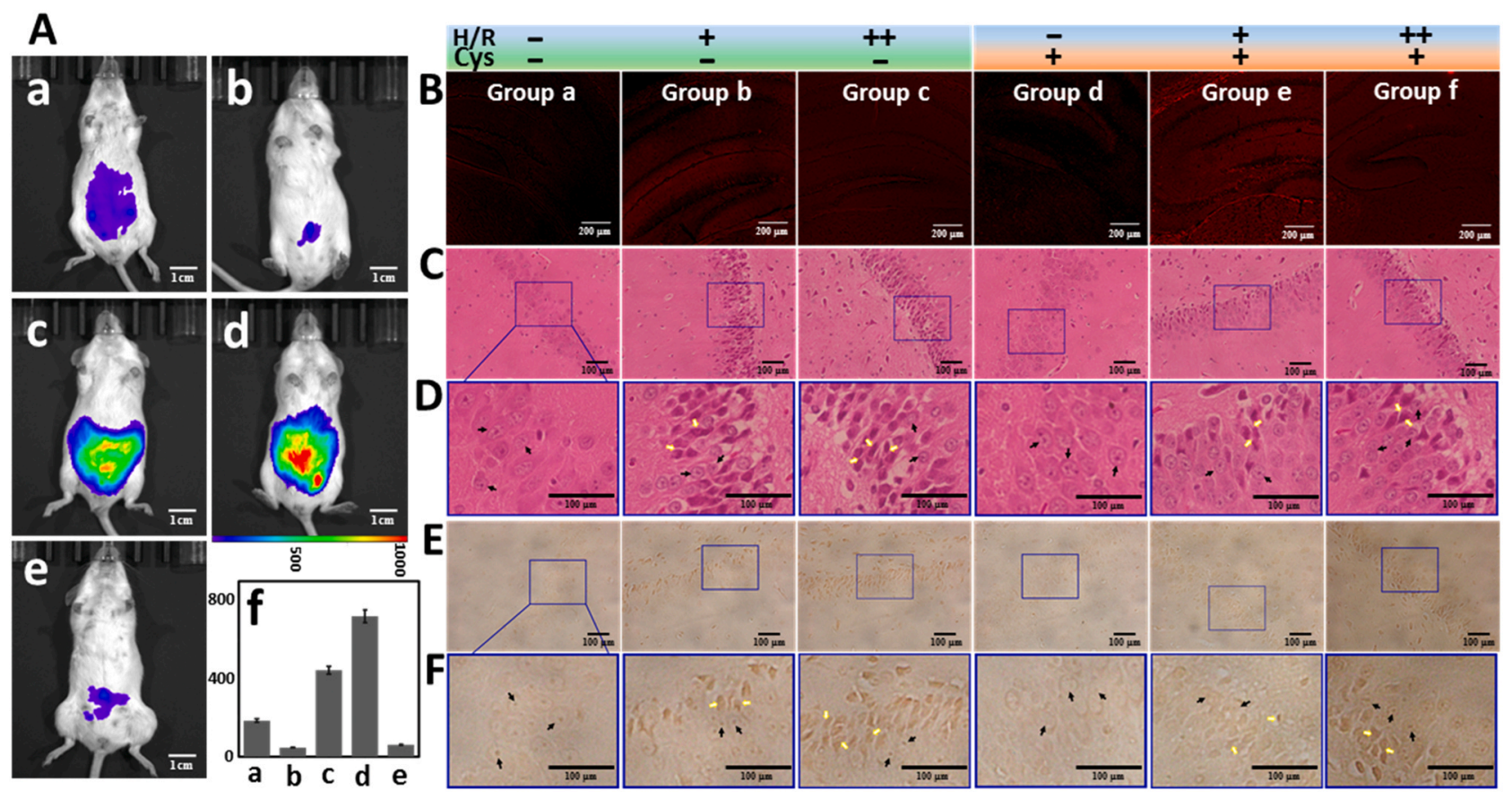

Fig. 6. (A) Fluorescence visualization of peritoneal cavity Cys levels via BCy-AC in BALB/c mice. a) The mice were i.p. injected probe (10 $\mu \mathrm{M}$, $100 \mu \mathrm{L}$ in DMSO/ saline, v/v 1:99) for $15 \mathrm{~min}$; b) The mice were first treated with NEM $(250 \mu \mathrm{g} / \mathrm{kg})$ for $1 \mathrm{~h}$, then stained BCy-AC; c)-d) Before imaging BCy-AC, the mice were pretreated with NEM and then incubated Cys $(100 \mu \mathrm{M}, 200 \mu \mathrm{L})$, Cys $(300 \mu \mathrm{M}, 200 \mu \mathrm{L})$ for 30 min, respectively; e) The mice were pretreated with NEM and then incubated Hcy $(300 \mu \mathrm{M}, 200 \mu \mathrm{L})$ for $30 \mathrm{~min}$; f) Quantification of fluorescence intensity in a)-e). Scale bar: $1 \mathrm{~cm}$. (B)-(F): The analysis of hippocampi slices in the H/R mice models and the evaluation of Cys efficacy for H/R damage. Confocal fluorescence imaging of endogenous Cys in hippocampi slices through BCy-AC (10 $\mu \mathrm{M})$ (B), scale bar: $200 \mu \mathrm{m}$; Representative slices of H\&E-stained hippocampi lesions tissue (C) and zooming views (D); Representative slices of TUNEL-stained lesions tissue (E) and zooming views (F), scale bar: $100 \mu \mathrm{m}$; The black arrows and yellow arrows indicate the normal and impaired cell states, respectively. The data were shown as mean ( \pm s.d.) $(n=6) . H / R+$ : hypoxia $1 \mathrm{~d} /$ reperfusion $2 \mathrm{~h}, \mathrm{H} / \mathrm{R}++$ : hypoxia $2 \mathrm{~d} /$ reperfusion $2 \mathrm{~h}$; Cys+: $100 \mu \mathrm{g} / \mathrm{kg}$ Cys in saline i.p. twice daily for 2 weeks before executed hypoxia. 
models were prepared to examine imaging performance of BCy-AC in tissue and evaluate Cys efficacy for the H/R stress (Fig. 6B-F). BALB/c mice were respectively placed in normobaric hypoxic chambers (FIO2, fraction of inspiration $\mathrm{O}_{2}, 11 \%$ ) for 1 day or 2 days to build different hypoxia models and then the hypoxia mice both performed $2 \mathrm{~h}$ reperfusion (Gao et al., 2018). The models were divided into two types, one was Cys-free (group a-c) and the other was pretreated with $100 \mu \mathrm{g} / \mathrm{kg}$ Cys twice daily for 2 weeks before executing H/R (group d-f). As shown in Fig. 6B, the Cys level in hippocampi of hypoxia 1 day/ reperfusion $2 \mathrm{~h}$ mice model (group b) was found higher than that of sham group (group a) through BCy-AC imaging. However, no matter in Cys-free groups or Cys-treated groups, the mice underwent 2 days hypoxia didn't exhibit more enhanced Cys levels in the hippocampi tissues (group c and f). The fluorescence of Cys levels in Cys-treated groups were slightly brighter than Cys-free groups. These results illustrated that BCy-AC was also suitable for observing endogenous Cys fluctuation in tissues and further investigating the relationship of Cys levels and H/R stress.

Considering the cerebral damage and possible impacts of Cys under mice H/R stress, the pathological sections of hippocampi tissue had been stained by H\&E and TUNEL in Fig. 6C and Fig. 6E. Combined with the enlarged view (Fig. 6D and Fig. 6F), the stained pathological slices exhibited that the cells in sham group were stained uniformly and arranged neatly without vacuolar; The cells arranged irregularly and accompanied by wrinkled nucleus, condensed cytoplasm, emerged vacuolar, contracted edges, and stained deeply in the both two $H / R$ models. In particular, the slices of hypoxia 2 days/ reperfusion $2 \mathrm{~h}$ (group c, f) displayed more serious cell injury than that of 1 day hypoxia and reperfusion model (group b, e). Under same H/R condition, the cell damage of two H/R models were widely alleviated when the mice pretreated Cys for 2 weeks, the number and degree of cellular deformation decreased, the vacuoles lessened, and the number of normal cells increased in the two H/R models. No matter in absence or presence of Cys pretreatment, there was no perceptible alteration between the control groups which illustrated that there was no distinction in results owing to Cys pretreatment (group a and group d). Endogenous Cys was up-regulated in hippocampi tissue during mice $H / R$ process and the damage was found alleviative when the mice pretreated with Cys for 2 weeks, which may be attributed to the antioxidant of Cys and its transformation with other RSS under H/R stress (Guo et al., 2012; Zhang et al., 2019a). The in situ visualization of BCy-AC for Cys tracking in mice reflected that the probe had good tissue penetrativity, combined with pathological analysis, it was found that Cys can resist the damage caused by $\mathrm{H} / \mathrm{R}$ environmental stress and potential application prospect of the probe in the future.

\section{Conclusions}

In summary, we have developed a NIR florescent probe BCy-AC for monitoring endogenous Cys as well as evaluating the biofunction of Cys under environmental hypoxia stress. Compared with traditional methods (such as MS, HPLC and HPLC-MS), BCy-AC in this work showed non-invasiveness, NIR fluorescence spectra $\left(\lambda_{\mathrm{ex}} / \lambda_{\mathrm{em}}=648 / 726 \mathrm{~nm}\right)$, tissue penetration, and in situ visualization. The unique Enol-Keto tautomerization of the probe boosted sensitivity and application via propelled reaction process, BCy-AC can be used not only to in situ monitor endogenous Cys in HT-22 cells, zebrafish and mice, but also as a supplementary tool for Cys assessment under environmental hypoxia stress. Using BCy-AC, we actually investigated Cys concentration changes in $\mathrm{H} /$ $R$ process and evaluated the connection between Cys concentrations and $\mathrm{H} / \mathrm{R}$-induced damage, and confirmed that Cys could play protective roles to defense environmental $\mathrm{H} / \mathrm{R}$ induced mitochondria related injury. Our work provided a new way for further studying the roles of endogenous reactive species in environmental stress induced pathological processes.

\section{CRediT authorship contribution statement}

Xia Zhang: Conceptualization, Methodology, Investigation, Formal analysis, Writing - original draft. Liangwei Zhang: Project administration, Validation, Writing - review \& editing. Xiaoyan Wang: Validation, Writing - review \& editing. Xiaoyue Han: Data curation, Investigation. Yan Huang: Funding acquisition, Writing - review \& editing. Bowei Li: Validation, Writing - review \& editing. Lingxin Chen: Supervision, Resources, Funding acquisition, Writing - review \& editing.

\section{Declaration of Competing Interest}

The authors declare that they have no known competing financial interests or personal relationships that could have appeared to influence the work reported in this paper.

\section{Acknowledgements}

This work was supported by the National Natural Science Foundation of China (Grant Nos. 21976209, 21778026, 22007005, 21804010), the Science and Technology Innovation Development Plan of Yantai of China (2020MSGY113), Shandong Provincial Natural Science Foundation Key Project (ZR2020KB022), the Program of Youth Innovation Promotion Association, CAS (Grant No. 2019217), and Taishan Scholar Project Special Funding (Grant No. ts20190962).

\section{Appendix A. Supporting information}

Supplementary data associated with this article can be found in the online version at doi:10.1016/j.jhazmat.2021.126476.

\section{References}

Cai, S., Liu, C., Jiao, X., Zhao, L., Zeng, X., 2020. A lysosome-targeted near-infrared fluorescent probe for imaging endogenous cysteine (Cys) in living cells. J. Mater. Chem. B 8 (11), 2269-2274.

Chen, X., Tian, X., Shin, I., Yoon, J., 2011. Fluorescent and luminescent probes for detection of reactive oxygen and nitrogen species. Chem. Soc. Rev. 40 (9), 4783-4804.

Cheng, D., Pan, Y., Wang, L., Zeng, Z., Yuan, L., Zhang, X., Chang, Y.T., 2017. Selective visualization of the endogenous peroxynitrite in an inflamed mouse model by a mitochondria-targetable two-photon ratiometric fluorescent probe. J. Am. Chem. Soc. 139 (1), 285-292.

Ebisch, I.M.W., Peters, W.H.M., Thomas, C.M.G., Wetzels, A.M.M., Peer, P.G.M., Steegers-Theunissen, R.P.M., 2006. Homocysteine, glutathione and related thiols affect fertility parameters in the (sub) fertile couple. Hum. Reprod. 21 (7), 1725-1733.

Gao, M., Wang, R., Yu, F., Li, B., Chen, L., 2018. Imaging of intracellular sulfane sulfur expression changes under hypoxic stress via a selenium-containing near-infrared fluorescent probe. J. Mater. Chem. B 6 (41), 6637-6645.

Gao, M., Zhang, X., Wang, Y., Liu, Q., Yu, F., Huang, Y., Chen, L., 2019. Sequential detection of superoxide anion and hydrogen polysulfides under hypoxic stress via a spectral-response-separated fluorescent probe functioned with a nitrobenzene derivative. Anal. Chem. 91 (12), 7774-7781.

Gao, W., Xu, K., Ji, L., Tang, B., 2011. Effect of gold nanoparticles on glutathione depletion-induced hydrogen peroxide generation and apoptosis in HL7702 cells. Toxicol. Lett. 205 (1), 86-95.

Gavrilescu, M., Demnerová, K., Aamand, J., Agathos, S., Fava, F., 2015. Emerging pollutants in the environment: present and future challenges in biomonitoring, ecological risks and bioremediation. New Biotechnol. 32 (1), 147-156.

Gray, J.S., Wu, R.S.S., Or, Y.Y., 2002. Effects of hypoxia and organic enrichment on the coastal marine environment. Mar. Ecol. Prog. Ser. 238, 249-279.

Guo, Z., Nam, S., Park, S., Yoon, J., 2012. A highly selective ratiometric near-infrared fluorescent cyanine sensor for cysteine with remarkable shift and its application in bioimaging. Chem. Sci. 3 (9), 2760-2765.

Guo, Z., Park, S., Yoon, J., Shin, I., 2014. Recent progress in the development of nearinfrared fluorescent probes for bioimaging applications. Chem. Soc. Rev. 43 (1), 16-29.

Han, X., Wang, R., Song, X., Yu, F., Chen, L., 2018a. Evaluation selenocysteine protective effect in carbon disulfide induced hepatitis with a mitochondrial targeting ratiometric near-infrared fluorescent probe. Anal. Chem. 90 (13), 8108-8115.

Han, X., Wang, R., Song, X., Yu, F., Lv, C., Chen, L., 2018b. A mitochondrial-targeting near-infrared fluorescent probe for bioimaging and evaluating endogenous superoxide anion changes during ischemia/reperfusion injury. Biomaterials 156, $134-146$. 
Jou, C.J., Spitzer, K.W., Tristani-Firouzi, M., 2010. Blebbistatin effectively uncouples the excitation-contraction process in zebrafish embryonic heart. Cell. Physiol. Biochem. 25 (4-5), 419-424.

Jung, H.S., Han, J.H., Pradhan, T., Kim, S., Lee, S.W., Sessler, J.L., Kim, J.S., 2012. A cysteine-selective fluorescent probe for the cellular detection of cysteine. Biomaterials 33 (3), 945-953.

Jung, H.S., Chen, X., Kim, J.S., Yoon, J., 2013. Recent progress in luminescent and colorimetric chemosensors for detection of thiols. Chem. Soc. Rev. 42 (14), 6019-6031.

Jung, H.S., Lee, J.H., Kim, K., Koo, S., Verwilst, P., Sessler, J.L., Kim, J.S., 2017. A mitochondria-targeted cryptocyanine-based photothermogenic photosensitizer. J. Am. Chem. Soc. 139 (29), 9972-9978.

Kim, G.H., Kim, J.E., Rhie, S.J., Yoon, S., 2015. The role of oxidative stress in neurodegenerative diseases. Exp. Neurobiol. 24 (4), 325-340.

Kimura, H., 2014. Hydrogen sulfide and polysulfides as biological mediators. Molecules 19 (10), 16146-16157.

Kimura, H., 2019. Signaling by hydrogen sulfide (H2S) and polysulfides (H2Sn) in the central nervous system. Neurochem. Int. 126, 118-125.

Kundu, K., Knight, S.F., Willett, N., Lee, S., Taylor, W.R., Murthy, N., 2009. Hydrocyanines: a class of fluorescent sensors that can image reactive oxygen species in cell culture, tissue, and in vivo. Angew. Chem. Int. Ed. 48 (2), 299-303.

Li, S.J., Fu, Y.J., Li, C.Y., Li, Y.F., Yi, L.H., Ou-Yang, J., 2017. A near-infrared fluorescent probe based on BODIPY derivative with high quantum yield for selective detection of exogenous and endogenous cysteine in biological samples. Anal. Chim. Acta 994, 73-81.

Liu, H., Radford, M.N., Yang, C.T., Chen, W., Xian, M., 2019. Inorganic hydrogen polysulfides: chemistry, chemical biology and detection. Br. J. Pharmacol. 176 (4), 616-627.

Liu, J., Liu, Y., Bu, W., Bu, J., Sun, Y., Du, J., Shi, J., 2014. Ultrasensitive nanosensors based on upconversion nanoparticles for selective hypoxia imaging in vivo upon near-infrared excitation. J. Am. Chem. Soc. 136 (27), 9701-9709.

Paulsen, C.E., Carroll, K.S., 2013. Cysteine-mediated redox signaling: chemistry, biology, and tools for discovery. Chem. Rev. 113 (7), 4633-4679.

Petrie, B., Barden, R., Kasprzyk-Hordern, B., 2015. A review on emerging contaminants in wastewaters and the environment: current knowledge, understudied areas and recommendations for future monitoring. Water Res. 72, 3-27.

Porter, A.G., Jänicke, R.U., 1999. Emerging roles of caspase-3 in apoptosis. Cell Death Differ. 6 (2), 99-104.

Ravichandiran, P., Prabakaran, D.S., Bella, A.P., Boguszewska-Czubara, A., Masłyk, M., Dineshkumar, K., Yoo, D.J., 2020. Naphthoquinone-dopamine linked colorimetric and fluorescence chemosensor for selective detection of $\mathrm{Sn} 2+$ ion in aqueous medium and its bio-imaging applications. ACS Sustain. Chem. Eng. 8 (29), 10947-10958.

Ravichandiran, P., Kaliannagounder, V.K., Bella, A.P., Boguszewska-Czubara, A., Masłyk, M., Kim, C.S., Yoo, D.J., 2021a. Simple colorimetric and fluorescence chemosensing probe for selective detection of $\mathrm{Sn} 2+$ ions in an aqueous solution: evaluation of the novel sensing mechanism and its bioimaging applications. Anal. Chem. 93, 801-811.

Ravichandiran, P., Prabakaran, D.S., Maroli, N., Boguszewska-Czubara, A., Masłyk, M., Kim, A.R., Yoo, D.J., 2021b. Mitochondria-targeted dual-channel colorimetric and fluorescence chemosensor for detection of $\mathrm{Sn} 2+$ ions in aqueous solution based on aggregation-induced emission and its bioimaging applications. J. Hazard. Mater. 415, 125593.

Reddie, K.G., Carroll, K.S., 2008. Expanding the functional diversity of proteins through cysteine oxidation. Curr. Opin. Chem. Biol. 12 (6), 746-754.

Rohlenova, K., Sachaphibulkij, K., Stursa, J., Bezawork-Geleta, A., Blecha, J., Endaya, B., Spacek, T., 2017. Selective disruption of respiratory supercomplexes as a new strategy to suppress Her2high breast cancer. Antioxid. Redox Signal. 26 (2), 84-103.

Sedgwick, A.C., Wu, L., Han, H.H., Bull, S.D., He, X.P., James, T.D., Yoon, J., 2018. Excited-state intramolecular proton-transfer (ESIPT) based fluorescence sensors and imaging agents. Chem. Soc. Rev. 47 (23), 8842-8880.

Shahrokhian, S., 2001. Lead phthalocyanine as a selective carrier for preparation of a cysteine-selective electrode. Anal. Chem. 73 (24), 5972-5978.

Sun, H., Liu, Y., Bai, X., Zhou, X., Zhou, H., Liu, S., Yan, B., 2018. Induction of oxidative stress and sensitization of cancer cells to paclitaxel by gold nanoparticles with different charge densities and hydrophobicities. J. Mater. Chem. B 6 (11), 1633-1639.

Wang, X., Li, P., Ding, Q., Wu, C., Zhang, W., Tang, B., 2019. Illuminating the function of the hydroxyl radical in the brains of mice with depression phenotypes by two-photon fluorescence imaging. Angew. Chem. 131 (14), 4722-4726.

Won, S.J., Kim, J.E., Cittolin-Santos, G.F., Swanson, R.A., 2015. Assessment at the singlecell level identifies neuronal glutathione depletion as both a cause and effect of ischemia-reperfusion oxidative stress. J. Neurosci. 35 (18), 7143-7152.

Xu, W., Teoh, C.L., Peng, J., Su, D., Yuan, L., Chang, Y.T., 2015. A mitochondria-targeted ratiometric fluorescent probe to monitor endogenously generated sulfur dioxide derivatives in living cells. Biomaterials 56, 1-9.

Xue, S., Ding, S., Zhai, Q., Zhang, H., Feng, G., 2015. A readily available colorimetric and near-infrared fluorescent turn-on probe for rapid and selective detection of cysteine in living cells. Biosens. Bioelectron. 68, 316-321.

Yang, X., Guo, Y., Strongin, R.M., 2011. Conjugate addition/cyclization sequence enables selective and simultaneous fluorescence detection of cysteine and homocysteine. Angew. Chem. 123 (45), 10878-10881.

Yin, C.X., Xiong, K.M., Huo, F.J., Salamanca, J.C., Strongin, R.M., 2017. Fluorescent probes with multiple binding sites for the discrimination of Cys, Hcy, and GSH. Angew. Chem. Int. Ed. 56 (43), 13188-13198.

Zhang, D., Chen, W., Kang, J., Ye, Y., Zhao, Y., Xian, M., 2014. Highly selective fluorescence off-on probes for biothiols and imaging in live cells. Org. Biomol. Chem. 12 (35), 6837-6841.

Zhang, J., Wang, J., Liu, J., Ning, L., Zhu, X., Yu, B., Zhang, H., 2015. Near-infrared and naked-eye fluorescence probe for direct and highly selective detection of cysteine and its application in living cells. Anal. Chem. 87 (9), 4856-4863.

Zhang, X., He, N., Huang, Y., Yu, F., Li, B., Lv, C., Chen, L., 2019a. Mitochondriatargeting near-infrared ratiometric fluorescent probe for selective imaging of cysteine in orthotopic lung cancer mice. Sens. Actuators B Chem. 282, 69-77.

Zhang, X., Huang, Y., Han, X., Wang, Y., Zhang, L., Chen, L., 2019b. Evaluating the protective effects of mitochondrial glutathione on cerebral ischemia/reperfusion injury via near-infrared fluorescence imaging. Anal. Chem. 91 (22), 14728-14736.

Zhang, X., Zhang, L., Gao, M., Wang, Y., Chen, L., 2020. A near-infrared fluorescent probe for observing thionitrous acid-mediated hydrogen polysulfides formation and fluctuation in cells and in vivo under hypoxia stress. J. Hazard. Mater. 396, 122673. 